\title{
Solid tumors are poroelastic solids with a chemo-mechanical feedback on growth
}

\author{
D. Ambrosi ${ }^{1}$, S. Pezzuto ${ }^{2}$, D. Riccobelli ${ }^{1}$, T. Stylianopoulos ${ }^{3}$, and P. Ciarletta ${ }^{1}$ \\ ${ }^{1}$ MOX-Dipartimento di Matematica, Politecnico di Milano, piazza Leonardo da Vinci 32, 20133 \\ Milano, Italy ${ }^{2}$ Universitá della Svizzera Italiana, Institute of Computational Science, Faculty of \\ Informatics, Via Giuseppe Buffi 13, 6900 Lugano, Switzerland ${ }^{3}$ Cancer Biophysics Laboratory, \\ Department of Mechanical and Manufacturing Engineering, University of Cyprus, P.O. Box 20537 \\ Nicosia 1678, Cyprus
}

\begin{abstract}
The experimental evidence that a feedback exists between growth and stress in tumors poses challenging questions. First, the rheological properties (the "constitutive equations") of aggregates of malignant cells are still a matter of debate. Secondly, the feedback law (the "growth law") that relates stress and mitotic-apoptotic rate is far to be identified. We address these questions on the basis of a theoretical analysis of in vitro and in vivo experiments that involve the growth of tumor spheroids. We show that solid tumors exhibit several mechanical features of a poroelastic material, where the cellular component behaves like an elastic solid. When the solid component of the spheroid is loaded at the boundary, the cellular aggregate grows up to an asymptotic volume that depends on the exerted compression. Residual stress shows up when solid tumors are radially cut, highlighting a peculiar tensional pattern. By a novel numerical approach we correlate the measured opening angle and the underlying residual stress in a sphere. The features of the mechanobiological system can be explained in terms of a feedback of mechanics on the cell proliferation rate as modulated by the availability of nutrient, that is radially damped by the balance between diffusion and consumption. The volumetric growth profiles and the pattern of residual stress can be theoretically reproduced assuming a dependence of the target stress on the concentration of nutrient which is specific of the malignant tissue.
\end{abstract}

\section{Introduction}

After Folkman \& Hochberg [1], the multicellar spheroid is a standard in vitro system used to evaluate the uncontrolled duplication rate of a tumor cell aggregate. A tumor spheroid is a cluster of cells floating in a culture medium, it is an ensemble of cells freely proliferating in an environment with large availability of nutrient. The malignant cells have lost the ability to self-regulate their own number through a normal apoptosis mechanism, regulated by the homeostasis with the environment; they duplicate in an uncontrolled manner, isotropically, producing a nearly spherical shape.

In the standard free-growth case, a plot of the diameter of the tumor vs. time typically exhibits an early stage of exponential growth, followed by a linear one. The transition from one regime to the other is mainly regulated by the availability of nutrient, that is driven by 
diffusion through the intercellular space. In fact, when the size of the tumor $R_{O}(t)$ is smaller than the typical diffusion length, the nutrient is everywhere available in the spheroid and the growth is volumetric [2]:

$$
\frac{d R_{o}^{3}}{d t} \simeq R_{o}^{3},
$$

so that $R_{O} \simeq e^{t}$. Conversely, when the diameter of the spheroid is much larger than the penetration length of the nutrient, one obtains surface growth, that is

$$
\frac{d R_{o}^{3}}{d t} \simeq R_{o}{ }^{2},
$$

and $R_{O} \simeq t$. In a realistic intermediate regime, the concentration of nutrients decays exponentially with the radius [3], favoring the external proliferation vs the internal one. This work is motivated by a number of recent experiments that demonstrate the dependence of the growth rate of a tumor spheroid on the mechanical load at the boundary. Some papers report a reduced apoptosis, with no significant changes in proliferation [4]. According to others, the cell division, rather than the cell death rate, is affected by stress [5]. To disentangle the puzzle of the biological feedback of stress on growth, we discuss first the rheology of the cellular aggregate as a living material, to point out its constitutive properties. We illustrate a number of arguments that support the hypothesis that a solid tumor is a poroelastic material, where the cells and the extra-cellular matrix represent the solid elastic component. A mathematical model based on such an assumption is able to predict inhomogeneities that can not be justified by fluid-like assumptions. In the last section we address the numerical simulation of the growth of a murine tumor. In vivo tumors reach a larger size, they can be partially vascularized, they have a more complex internal composition and exhibit release of residual stress. We test the ability of our mathematical model comparing the observed and the predicted opening angle after excision.

\section{Background: elementary rheology and growth theory}

The simplest distinction among fluid and solid materials can be based on an elementary ideal experiment: under a pure shear load fluids flow, while solids do not, at the time scale of interest. This draconian categorization encompasses also viscoelastic materials, as they typically exhibit fluid-like or solid-like properties depending on the relaxation time scales. As an example, a "Maxwell fluid" behaves as a solid if observed at a time scale much smaller than its relaxation time. Analogously, a "Kelvin solid" flows like a fluid when observed on short enough time scales. Things become a little bit more complex when flow is prompted only above a yield stress, but the distinction persists when loads are neatly below or beyond the threshold.

Many biological materials are composed by a mixture of several components: interstitial fluid, different species of cells, collagen fibres, and so on. For these microscopically heterogeneous materials the overall mechanical behavior is represented, at the macroscale, 
by the superposition of single phase contributions, proportionally to the volume fraction occupied by each component. The archetypical example of a mixture is a porous elastic material permeated by a fluid: the stress in a poroelastic medium is the sum of the interstitial pressure of the fluid plus the solid stress, which is proportional to the solid volume fraction.

Fluids and solids behave in a very different manner when internal stresses arise not because of external loads, but as due to the inner material reorganization (growth and remodelling). The simplest example are thermal stresses in inhomogeneously heated materials with temperature-dependent density: residual stresses relax in fluids, not in solids. The persistence of residual stress is therefore the signature of solid-like behavior which has to be properly addressed in a modelling framework. In case of small strains, linearized elasticity applies and stress (and strains) can be superimposed. In case of large strains, as it is often the case with soft matter, a multiplicative decomposition of the tensor gradient of deformation has to be introduced.

For our purposes, we represent the motion of every material point of a continuous body as a smooth invertible map $\chi(\mathbf{X})$ with Jacobian $\mathrm{F}=\frac{\partial \chi}{\partial \mathrm{X}}$ For a nonlinear elastic material the strain energy is $W(\mathrm{~F})$; when the body grows and residual stress is present, the strain energy rewrites

$$
W\left(\mathrm{FG}^{-1}\right)
$$

where $\mathbf{G}$ is usually called "growth tensor".

\section{Are solid tumors fluids?}

While the availability of nutrients is the major factor affecting tumor growth, other external agents can play a role. The mechanical influence of external loading on tumor growth has been first demonstrated by Helmlinger et al. [4]. They designed an experimental setup in order to control the load applied at the boundary of tumor cell spheroids in vitro in agarose gels, and checked the influence of such a stressed state on the growth rate of the multicell spheroid. They compared the free growth of a floating multicell spheroid with the size of cell aggregates placed into the agarose gel. The gel is produced at a given (known) stiffness by suitably tuning the concentration of the solid phase. As the spheroid grows, it displaces the surrounding gel, which then exerts a compressive force at the surface of the tumor spheroid. An a priori mechanical characterization of the gel allows to calculate the pressure exerted by the gel on the spheroid, depending on its radius.

The main result of the experiments carried out by the group of Rakesh Jain [4] is that the stress field reduces the final size of the spheroids, with a decreased apoptosis and non significant changes in proliferation. It is therefore clear that a precise determination of the constitutive laws that characterize the mechanical behavior of a tumour spheroid is a prerequisite in order to assess a reliable stress-growth relationship. 
Early attempts in this respect assumed that a cell conglomerate behaves like a viscoelastic fluid, able to bear a static load because of its surface tension [6]. At equilibrium, measurements of the curvature radius of a loaded sample provide the surface tension of the "fluid".

According to the Laplace formula, the pressure jump across a curved interface between two fluids is inversely proportional to the radius of the curvature. If the spheroid is loaded with the force $F$ acting on a contact surface $A$, by continuity of the stress, the inner pressure is $F / A$ and therefore

$$
\frac{F}{A}=\sigma\left(\frac{1}{R_{1}}+\frac{1}{R_{2}}\right)
$$

where $\sigma$ is the surface tension and $R_{1}, R_{2}$ are the curvature radii of the free surface. According to the experiments, the surface tension of a cell aggregate ranges in $1-22 \cdot 10^{-3}$ Newton/meter (as a reference value, the surface tension of the water is about $72 \cdot 10^{-3}$ Newton/meter). Relaxation times range between 1 and 50 seconds [6].

The opposite approach is to describe a solid tumour as a viscoelastic solid. In this case, at equilibrium the external load should be balanced by the stress in the body, depending on the strain of its material points. Assuming an homogeneous deformation and using the same data provided by the experiments above, one can estimate the Young modulus $E$ according to the following rule:

$$
\frac{F}{A}=E \frac{h-h_{0}}{h_{0}}
$$

where $h, h_{0}$ are the height of the loaded and unloaded sample, respectively. In this case one finds $E \simeq 4 \mathrm{kPa}$, a typical soft-range value for living cells [7].

A second argument supporting the assumption of solid-like constitutive equations is based on the spatial correlation between stress and apoptosis-mitosis in loaded ellipsoidal spheroids [8]. The non-homogeneous proliferation pattern can be produced only by a solidlike material: a hydrostatic generates a pressure independent on the position in any symmetric geometry, while in a solid material, high stress concentrates around the tips.

Furthermore, the work by Netti et al. [9] support the view that tumors behave as solid-like materials. In their study, stress-relaxation experiments of various tumor types in confined compression were performed and at the end of the experiment all tumors equilibrated in a constant, non-zero stress, typical of viscoelastic solids.

Finally, evidence of residual stress in murine and human tumors is reported by Stylianopoulos et al [10]. They cut the tumor azimuthally and observed an opening angle, which is the signature of a solid like behavior. Residual stress is likely produced by an inhomogeneous duplication rate of the cells as well as by mechanical interactions between 
the cells and extracellular matrix components, particularly collagen and hyaluronic acid, that strain the tumor microenvironment. Only solids can contain residual stress, due to the evolution of their relaxed configuration produced by incompatible growth [11]: energy can be elastically stored in the unloaded body only if it is a solid. In particular, Stylianopoulos et al. observed a compressive residual stress (i.e. negative opening angle) in the kernel and a tensile residual stress (positive opening angle) in the outer shell of the tumor. This behaviour is paradoxical in terms of availability of nutrients: their concentration is larger near the boundary, thus favoring proliferation and eventually producing compressive stress. In a solid tumor in vivo, this intuitive explanation does not work and we address such a puzzle in the next sections.

\section{Growth and stress}

An evocative definition of a tumor is "a living system that has lost its self-regulating ability towards homeostasis". In other words, tumor cells do not correctly detect or elaborate the external signals that should regulate its proliferation and apoptosis, and duplicate without control. When the stress state of the system is not in homeostatic mechanical equilibrium, it remodels (growing or resorbing matter) until the target tensional state is recovered. In this respect, all the genetic information that detail the shape and function of organs are encoded in the target stress. A suggestive mechanical interpretation of a tumor therefore naturally arises: a tumor is an open system (in terms of mass and energy) with a damaged inner mechano-biological control inducing a disregulation of tensional homeostatis, i.e. the feedback that normally self-regulates growth in terms of stress-modulated control does not properly work. In other words, tumor cells regulate production and consumption not according to a benefit of the whole organism but only in view of maximum invasion of malignant cells: the control on growth does indeed exist, also as a function of available nutrients, but the corresponding duplication/apoptosis strategy has a different aim.

The experiments illustrated in the section above do not only demonstrate the existence of residual stress in tumors, but they also show that the inhomogeneous proliferation and apoptosis, triggered by the differential availability of nutrients, is enhanced in a mechanically loaded spheroid. Their main result is that mechanical stress affects proliferation and apoptosis inside the spheroid in a non-homogeneous way, a correlation existing between strong apoptosis and high stress.

In another series of experiments, the compression of the spheroid is controlled by the concentration of a large molecule (Dextran) soluted in the bath [12,5]. As Dextran molecules cannot enter neither the cell membrane nor the interstitial (intracellular) space, an imbalance of osmotic pressure at the boundary loads the cellular aggregate. It is reported that for larger concentrations of Dextran the diameter of the spheroid grows slower and reaches a plateau at smaller radius, in agreement with the results of Helmlinger et al [4]. While a single cell is almost incompressible with respect to the pressure due to the concentration of Dextran, the volume of the cell aggregate strongly depends on the osmotic pressure [13]. The reduction in volume in the cellular aggregate therefore mainly occurs because of reduction of the intercellular space, in the inner region of the spheroid. 
The large number of available data suggests that a cell aggregate behaves as a poroelastic material. The mathematical modelling of solid tumors as porous deformable media has been addressed in a number of papers $[14,15,16]$; it a suitable mechanical framework to account for the coupled dynamics of cells and extracellular matrix (the solid matrix) and interstitial fluid. The interstitial flow is typically represented by a Darcy-type equation, while the mass exchange among phases which allows a prediction of the growth of the mass.

In the experimental setup by Montel et al. $[12,5]$ the porous media theory offers a transparent explanation for interplay between the pressure of the fluid, the chemical potential of the Dextran and the stress in the solid matrix. The external load at the boundary is the sum of two terms: the pressure of the fluid plus the chemical potential of the Dextran. Observing that the diameter of the macromolecules is typically larger than the size of the intracellular pores, we split the fluid load into two contributions: one that balances the interstitial pressure, the other one loading the solid (cellular) component. Formally, we assume that the global balance at the boundary

$$
\left(-p-p_{D}\right)_{\text {out }} \mathbf{n}=(\mathbf{T}-p \mathrm{I})_{\text {in }} \mathbf{n},
$$

splits into

$$
\begin{gathered}
-p_{\text {out }}=-p_{\text {in }}, \\
-p_{D} \mathbf{n}=\mathbf{T n} .
\end{gathered}
$$

where $p$ is the pressure of the interstitial fluid, $p_{D}$ is the osmotic pressure contribution due to the concentration of Dextran, $\mathbf{T}$ is the Cauchy stress tensor in the cellular aggregate, $\mathbf{I}$ is the identity tensor and $\mathbf{n}$ is the outgoing normal (radially directed) vector. This assumption is in agreement with the observation that the solid stress is not affected by the interstitial fluid pressure [10].

On the basis of this hypothesis, the stress state in the loaded spheroid can be determined solving the force balance equations for the solid component only. Assuming spherical symmetry, the tensor gradient of deformation and the growth tensor read

$$
\mathrm{F}=\operatorname{diag}\left(r^{\prime}, \frac{r}{R}, \frac{r}{R}\right), \quad \mathrm{G}=g(r) \mathrm{I},
$$

where $r(R, t)$ is the radial coordinate of the material point that was in $R$ at time $t=0, \mathbf{I}$ is the identity tensor and the prime ' denotes derivation in $R$. The solid component of the poroelastic spheroid must satisfy the force balance equation 


$$
\frac{d}{d r} T_{r r}+\frac{2}{r}\left(T_{r r}-T_{\theta \theta}\right)=0,
$$

with boundary conditions

$$
r(0, t)=0, \quad T_{r r}\left(r_{o}\right)=-p_{D} .
$$

where $r_{O}=r\left(R_{O}, t\right)$. A simple representation of an hyperelastic compressible material is provided by the strain energy

$$
\hat{W}(\mathrm{~F})=\frac{\mu}{2}(\mathrm{~F} \cdot \mathrm{F}-2 \log (\operatorname{det} \mathrm{F})-3) .
$$

If the material grows, the strain energy depends on the growth tensor too, through a classical multiplicative decomposition

$$
W\left(\mathrm{FG}^{-1}\right)=\frac{\mu}{2}\left(\mathrm{FG}^{-1} \cdot \mathrm{FG}^{-1}-2 \log \left(\operatorname{det}\left(\mathrm{FG}^{-1}\right)\right)-3\right) .
$$

where $\mu$ is the shear elastic modulus. First variation and pull back to the reference configuration yields the first Piola-Kirchhoff stress

$$
\mathrm{P}=\mu \operatorname{det}(\mathrm{G})\left(\mathrm{FG}^{-1} \mathrm{G}^{-T}-\mathrm{F}^{-T}\right)
$$

where, explicitly,

$$
P_{R R}=\mu g^{3}\left(\frac{r^{\prime}}{g^{2}}-\frac{1}{r^{\prime}}\right), \quad P_{\Theta \Theta}=\mu g^{3}\left(\frac{r}{R g^{2}}-\frac{R}{r}\right) .
$$

The force balance equation (10) in material coordinates reads

$$
\frac{d}{d R} P_{R R}+\frac{2}{R}\left(P_{R R}-P_{\Theta \Theta}\right)=0
$$

or, explicitly,

$$
\frac{d}{d R}\left(r^{\prime} g-\frac{g^{3}}{r^{\prime}}\right)=2\left(g \frac{r}{R^{2}}-\frac{g^{3}}{r}-g \frac{r^{\prime}}{R}+\frac{g^{3}}{r^{\prime} R}\right)
$$


to be supplemented by boundary conditions (11) rewritten in material coordinates

$$
P_{R R}\left(R_{O}\right)=\left.(\operatorname{det} \mathrm{F}) T_{r r} F_{r r}^{-1}\right|_{R_{0}}=-\left.(\operatorname{det} \mathrm{F}) p_{D} F_{r r}^{-1}\right|_{R_{0}}
$$

or, explicitly

$$
\left.\mu g^{3}\left(\frac{r^{\prime}}{g^{2}}-\frac{1}{r^{\prime}}\right)\right|_{R_{0}}=-\left.p_{D} \frac{r^{2}}{R^{2}}\right|_{R_{0}} .
$$

For constant $g$ the force balance equation (17) with boundary conditions (19) has solution

$$
r(R ; g)=\gamma g R
$$

where $\gamma$ is the positive root of the third order polynomial

$$
f(\gamma)=p_{D} \gamma^{3}+\mu \gamma^{2}-\mu=0
$$

One may notice that $f(0)=-\mu<0$ while $f^{\prime}$ is always positive, therefore the root is unique. Moreover $f(1)=p_{D}>0$, so that it must be $0<\gamma<1$.

\section{Remark}

One could observe that poroelasticity has been advocated for the model above, but its use is apparently very limited: there is no interstitial fluid flow, and the porosity, the volume fraction of solid vs. liquid component, is not even mentioned. There is a rationale behind such a minimal choice. Fluid flow is so slow that it carries no contribution in the stress balance equation; of course, mass exchange among species is the true physical mechanism for the growth of the tumor mass, however here it is directly incorporated in the growth tensor $\mathbf{G}$. Secondly, the porosity of the matrix should contribute to the stress tensor $\mathbf{T}$ with a multiplicative factor depending on the determinant of the gradient of deformation; as a matter of fact, we incorporate such a contribution in the compressibility of the strain energy function (12). The numerical results to be illustrated in the next sections will confirm that good predictions can be obtained even with such a simple constitutive law, thus confirming that the theory weakly depends on the specific constitutive equation for the strain energy density of the solid matrix. The crucial ingredient of the model is the multiphase split of the load at the boundary into a fluid and a solid component (9).

\section{Mechanobiological feedback and equilibrium}

In the general case, a growth law for $\mathbf{G}$ is to be supplemented to close the differential equation (17-19). We consider first the case of growth controlled by a mechanical feedback only. If nutrients are largely available everywhere, the growth in time is expected to depend on the stress only. In finite elasticity, the growth must depend on an invariant measure of the 
stress. A thermodynamically consistent choice is to adopt the dependence on the Eshelby stress [17]. To minimize the calculations, while preserving the essential biophysical features, we chose here to measure the stress in terms of the second Piola-Kirchhoff tensor $\mathbf{S}$, which reads

$$
\mathrm{S}=\mathrm{F}^{-1} \mathrm{P}
$$

The mitotic rate of single tumor cells is known to be inhibited by compression [4], and promoted by tension [18], and a very simple growth law that can account for such a behavior is

$$
\dot{g}=\frac{g}{\tau}\left(1+\frac{\operatorname{tr} S}{3 \kappa \mu}-\frac{g}{\alpha}\right)
$$

where $1 / \tau$ is the mitotic rate in absence of external stimuli, $\kappa \mu$ is a threshold stress and the last term in brackets accounts for apoptosis, the natural cellular death rate. We highlight that the assumption of an isotropic growth tensor allows to set a functional dependence on the trace of $\mathbf{S}$. For a general anisotropic growth a more complex dependence on the principal stresses would be needed, guided by thermo-mechanical requirements.

Consider the unloaded case first: $-p_{D}=0$ and at time $t=0$ the solid component has $g=1$. As $\mathbf{S}=0$ the evolution in time of $\mathbf{G}$ is autonomous and independent of the radial position, so that $g(t)$ is constant in space and its evolution in time initially follows the well known exponential growth in size of the cell aggregate up to a saturation dictated by the value of $a$. The solid component of the poroelastic spheroid is therefore relaxed, exactly as a sponge in the deep ocean, where the interstitial pressure balances the head of the water.

If Dextran is present, the extra pressure compresses the cellular phase and triggers the mechanobiological feedback via equations (23). The growth $g(t)$ is given by the solution of the first order ordinary differential equation

$$
\dot{g}=\frac{g}{\tau}\left(1-g\left(\frac{\gamma p_{D}}{\kappa \mu}+\frac{1}{\alpha}\right)\right)
$$

Equation (24) has two equilibrium points: $g=0$, always unstable, and

$$
g_{e}=\frac{\alpha \kappa \mu}{\alpha \gamma p_{D}+\kappa \mu}
$$

which is always stable (for the fixed $r(R ; t)$ of $(20)$ ). The mathematical model therefore predicts the following scenario, corresponding to the observed dynamics. For null osmotic pressure, the system grows exponentially, then it tends to saturation. For sufficiently large 
osmotic pressure the stable equilibrium depends on the applied pressure $p_{D}$. After derivation of equations (21) and (25) we get

$$
\frac{d g_{e}}{d p_{D}}=-\frac{\alpha^{2} \kappa \mu}{\left(\alpha \gamma p_{D}+\kappa \mu\right)^{2}} \frac{\gamma\left(2 \gamma p_{D}+2 \mu\right)}{3 \gamma p_{D}+2 \mu}<0
$$

The solution of equation (24) explains the plateau in growth vs time reported for loaded spheroids at different Dextran concentrations, but it does not account for the radial density inhomogeneities observed in excised aggregates. Remaining in a purely mechanical setting, an explanation for such a discrepancy between theory and experiments could be provided by the possible onset of an instability for the equilibrium solution (24) of the coupled problem. This question is addressed in the appendix, where we study the stability of the solution of the nonlinear system (17) and (23) with boundary conditions (19) in order to explain the emergence of inhomogeneity. The result of the analysis is that the small perturbations are always damped in time, so that a purely mechanical framework cannot account for the observed dependency of growth on the radial coordinate. The biophysics of the system needs therefore to be enriched: in the next section we show that the kinetics of nutrients can trigger dependence of the asymptotic state on the radial coordinate.

\section{Dynamics of the nutrient and inhomogeneity of growth}

In an avascular tumor, nutrients are provided to malignant cells by diffusion through the boundary of the spheroid. The balance between diffusion and uptake is fast with respect to the growth times (one hour vs. days) and obeys a linear reaction-diffusion equation:

$$
-\frac{1}{r^{2}} \frac{d}{d r}\left(r^{2} \frac{d c}{d r}\right)=-\frac{c}{\lambda^{2}} .
$$

with boundary conditions

$$
\left.\frac{d c}{d r}\right|_{r=0}=0, \quad c\left(r_{0}\right)=c_{0},
$$

where the decay length $\lambda$ is on the order of 100-200 micrometers and $c_{0}$ is the external (constant) concentration. We remind that the boundary value problems refers to the avascular phase of tumor growth. At later stages, neovascularization can be triggered after the diffusion-limited radius is reached. In such a case, a distributed nutrient supply from the tumor vascular network should also be taken into consideration.

During the avascular growth phase, the concentration profile can be calculated by direct integration of the equation in spatial coordinates [3], yielding an exponential decay of the concentration of nutrient going from the boundary to the center of the spheroid: 


$$
c(r)=c_{0} \frac{r_{0}}{\sinh \left(r_{0} / \lambda\right)} \frac{\sinh (r / \lambda)}{r} .
$$

To account for the combined action of stress and nutrient pattern, we propose to rephrase equation (24) to the following growth law

$$
\dot{g}=\frac{g}{\tau} c\left(1+c\left(\frac{\operatorname{tr} S}{3 \kappa \mu}-\frac{g}{\alpha}\right)\right) .
$$

According to equation (30), the proliferation of the malignant cells is enhanced by the availability of nutrient, as it is usually assumed in mathematical models that do not specifically account for mechanics. In the same way as in equation (23), it is expected that the system reaches an equilibrium when the term in brackets vanishes: a plateau in size is observed for large enough times. The novelty of this growth law is that the equilibrium does not correspond to an homogeneous growth tensor $g \mathbf{I}$, but it depends on the radial position through the concentration of nutrient, thus originating an inhomogeneous residual stress. Using numerical simulations, in the next sections we are able to show that the predicted residual stress is in agreement with the reported opening angles from cutting experiments.

\section{Numerical simulations}

Numerical integration of equations (17) and (30) with boundary conditions (19) and initial conditions

$$
r(R, t=0)=R, \quad g(R, t=0)=1
$$

is performed using a finite difference scheme with centered discretization in space and a fourth order Runge-Kutta scheme in time. The parameters used in the numerical simulations are $\tau=2.5$ days, $\kappa=2.9 \mathrm{kPa}, a=3.7, \lambda=250 \mu \mathrm{m}$ and $\mu=10 \mathrm{kPa}$. The initial radius is 100 $\mu \mathrm{m}$, the final simulation time is $t_{f}=25$ days and the boundary condition of (27) is $c_{0}=1$.

The volume of the spheroid initially grows very rapidly for all values of $p_{D}$. At large times, for null or small values of osmotic pressure the slope of the curve becomes very small, and it becomes horizontal for large $p_{D}$ (Figure 1).

As expected, the non-uniform pattern of nutrients triggers a weak inhomogeneity in growth. While the predicted growth pattern cannot be directly compared with data, it is indirectly supported by the residual stress that it produces by the relation (3). The radial and hoop component of the residual stress are plotted in Figures 2 and 3 versus the radial coordinate at equilibrium.

As expected, the radial stress vanishes on the boundary of the spheroid, while it is internally compressive. Conversely, the hoop stress changes sign, being compressive in the core and 
tensional in the outer layer. Such a residual stress distribution is stable against both circumferential and azimuthal perturbations of the tumour boundary, as investigated in [19].

Data on residual stress of in vitro tumor spheroids are not available, probably because they are too soft and do not reach a size such that a mechanical manipulation and a precise cut can be operated. However the pattern reported in Figure 2 and Figure 3 is in qualitative agreement with experiments on (much bigger) human tumors implanted in mice [10]. Stylianopoulos et al. observe that cells at the periphery of the spheroid are restricted by the surrounding tissue and thus, during radial tumor growth they develop tensile circumferential forces. Surrounding tissues in vivo would then produce on the tumor a compressive hydrostatic pressure increasing with the tumour growth. Furthermore, Figures 3 and 4 depict that the magnitude of stress - either compressive or tensile - increases as the osmotic pressure exerted on the cells, $p_{D}$, decreases. This is explained by the fact that for low osmotic pressures the tumor becomes larger in size and the stresses increase.

If the external pressure is removed, the radius quickly grows and reaches the same value of the free-growth case (Figure 4), in agreement with the experimental results [12].

\subsection{Stress release in a cut spheroid}

A quantitative comparison among observed and predicted residual stress can be obtained on the basis of the opening angle of cut specimens. To this aim, tumor spheroids have been grown in mice and then they have been cut along their azimuthal plane for about $80 \%$ of their diameter. The spheroids then partially relax their residual stress: the cut surface opens up at the periphery while the inner region swells (see figure 5). Figure 6 depicts the cutting experiments for breast and pancreatic tumors implanted in nude mice, also reporting the tumor opening length and the maximum residual stresses within the tumor specimens.

The observed behavior, which is in qualitative agreement with our predictions in the stress pattern in small, in vitro, spheroids, can be quantitatively compared with opening angles data on the basis of a three dimensional numerical simulation only. As a matter of fact, an axial cut of a ring preserves the cylindrical symmetry of the problem [20], while an azimuthal cut of a sphere breaks it.

Numerical simulations are obtained using a finite element code that solves the equation of finite elasticity on a spherical wedge. The computation reproduces the physical observations: the spheroid grows under spherical symmetry which is eventually broken by the cut. We therefore use the growth tensor computed under radial symmetry assumption and we evaluate the opening angle that it produces.

The 3D numerical problem is based on FEniCS [21]. The computational domain is discretized with quadratic tetrahedral elements, with an average diameter of $10 \mu \mathrm{m}$. Since we expect near to singular stresses around the edge of the cut, the mesh is gradually refined nearby this edge to one twentieth of the original size. The mesh contains roughly 28143 elements and it has been produced by Gmsh [22]. The non-linear variational problem is discretized with quadratic isoparametric finite elements, and the final problem has 137949 degrees of freedom. The solver for non-linear problem is based on a modified Newton's 
method specifically designed for variational inequalities, and implemented in the PETSc framework [23]. The solver can deal with inequality constraints, as we have on the cut boundary surface to avoid self-contact during the swelling. The solver for the linear system is MUMPS [24].

The simulation is performed in two steps: first, we apply a homogeneous growth tensor obtained by averaging the target one, while keeping the cut sealed; then, we release the cut and we enforce the final growth tensor. This strategy facilitates the convergence of the nonlinear solver, which performs 40 iterations at most. A relative error below $1 \%$ on the opening angle is observed when the mesh is uniformly refined, certificating the numerical convergence.

Boundary conditions, reported in Figure 7, apply as follows: the outer boundary is stressfree, the cut surface is enforced to have non-negative displacement in the normal direction to avoid self-contact, and on the internal, intact, portion of the boundary symmetry arguments yield null normal displacement and while the other components of the displacement must have null derivative with respect to the normal direction. In Figure 8 the deformed configuration obtained after a vertical cut of $80 \%$ of the diameter is shown.

The parameters used in the numerical simulations are $\tau=2.5$ days, $\kappa=33.35, a=37, \lambda=$ $2.5 \mathrm{~mm}, \mu=27.0 \mathrm{kPa}$ and $p_{D}=5.0 \mathrm{kPa}$. The initial radius is $100 \mu \mathrm{m}$, the simulation ends at $t$ $=50$ days and the boundary condition of (27) is $c_{0}=1$. In the numerical experiment the tumor opens with an angle of $11.70^{\circ}$, corresponding to $1.41 \mathrm{~mm}$ of opening length. The final volume is $169.84 \mathrm{~mm}^{3}$. The inner-most part of the cut, for about $20 \%$ of the diameter, is in self-contact, certificating that this portion of the tumor tends to swell outward after the cut. It is to be remarked that the diffusion length assumed here is larger than the one used for small in vitro spheroids.

These results are in agreement with the ex-vivo experiments: the opening length, the final volume and the hoop stress are very close to the reported ones for the MiaPaCa2 tumor number 4 (see Figure 6) [10].

In order to investigate the relationship between the heterogeneity in the growth tensor and the opening angle, we have performed a numerical experiment where the difference between the growth at the center and the boundary of the tumor is stepwise increased from zero to a value of 20. Table 1 summarizes the result of the simulation. As expected from the theory, a uniform growth yields no residual stress and the tumor does not open after the cut (first column of the table). On the other hand, the greater the difference in growth between the center and the boundary, the larger the opening length and consequently the opening angle (from the second column of the table). The volume is mostly affected by the average value of the growth over the entire domain, and not by the heterogeneity. The numerical experiment also shows that the angle linearly increases with the difference in growth of about $8^{\circ}$ every 10 units per mm of growth. 


\section{Final remarks}

The growth of a tumor spheroid can be controlled using mechanical stress: when an osmotic pressure is applied at the boundary, the radius of the aggregate grows in time until it reaches an equilibrium volume which inversely depends on the load. The size control is fully reversible: when traction is released, the cellular matrix relaxes and returns the original growth curve. The observation that the intercellular space forms a pore-like structure, that macromolecules cannot enter, suggests to represent mechanically the cellular aggregate as a poroelastic material [25]. The evidence of a residual stress leads to the assumption that the solid phase is hyperelastic: the large compliance of the cell aggregate is due to the squeezing of the intracellular fluid and the corresponding reduction of the intracellular space, while the single cells are much stiffer [13]. Boundary conditions are split accordingly: the osmotic pressure generated by the Dextran solution of the surrounding fluid loads the solid phase only.

The exponential decay in the pattern of nutrients makes the proliferation process of a sufficiently large loaded spheroid inhomogeneous and the generated residual stress depends on the radial position: it is compressive near the center and tensional at the periphery [10]. This feature is paradoxical when compared with the usual scenario: large availability of nutrients at the periphery of the spheroid is expected to favour the proliferation and, therefore, emergence of compressive residual stress. The dynamics of tumor growth is apparently different: tumor cells duplicate (or control their apoptosis) on the basis of the available nutrients, but their target stress modulates so as to produce compression in regions with small concentration of nutrients. In other words, the reported radial distribution of residual stress can be explained only admitting that in the inner regions, where the concentration of nutrients is very small, malignant cells slow down their apoptotic rate, in agreement with the observations of Helmlinger et al [4].

After a standard multiplicative decomposition of the tensor gradient of deformation to account for growth, we introduce a simple law of biomechanical feedback, and we are eventually able to explain the observed dynamics. On the basis of such a conjecture, we were able to reproduce growth profiles of tumor spheroids for different values of the applied load and the open angles of mice bearing breast tumors. Particularly for the second result, the solid phase of mice bearing tumors apart from cells consists also of the extra-cellular matrix, which can contribute to the development of residual stress. Here the mechanical contribution of all components of the tumor are resumed in the solid phase, and the growth tensor $\mathbf{G}$ accounts also for the possible tensional contribution due to the elongation of the collagen fibres.

Our mechanobiological model explains the observed smaller asymptotic volume as a function of increasing osmotic load on the basis of a stress-growth coupling. At later stages, not covered by the present model, when the radial inhomogeneity is fully developed, the solid (cellular) component of the spheroid undergoes a stress per volume fraction larger than a threshold that takes it into the plastic regime [26]; then cells start flowing centripetally, producing an internalization of the cells from the periphery to the center of the tumor [27, 28]. 


\section{Supplementary Material}

Refer to Web version on PubMed Central for supplementary material.

\section{Acknowledgments}

The work has been partially supported by the My First AIRC Grant - MFAG 2015 - code 17412 titled "A Mathematical insights of glioblastoma growth: a mechano-biology approach for patient-specific clinical tools" and by "Progetto Giovani GNFM 2016" funded by the National Group of Mathematical Physics (GNFM - INdAM). TS is supported by European Research Council (ERC-2013-StG-336839).

\section{References}

[1]. Folkman J, Hochberg M. Self-regulation of growth in three dimensions. The Journal of experimental medicine. 1973; 138(4):745-753. [PubMed: 4744009]

[2]. Ambrosi D, Mollica F. The role of stress in the growth of a multicell spheroid. Journal of mathematical biology. 2004; 48(5):477-499. [PubMed: 15133619]

[3]. Greenspan H. Models for the growth of a solid tumor by diffusion. Stud Appl Math. 1972; 51(4): 317-340.

[4]. Helmlinger G, Netti PA, Lichtenbeld HC, Melder RJ, Jain RK. Solid stress inhibits the growth of multicellular tumor spheroids. Nature biotechnology. 1997; 15(8):778-783.

[5]. Montel F, Delarue M, Elgeti J, Vignjevic D, Cappello G, Prost J. Isotropic stress reduces cell proliferation in tumor spheroids. New Journal of Physics. 2012; 14(5):055 008.

[6]. Forgacs G, Foty RA, Shafrir Y, Steinberg MS. Viscoelastic properties of living embryonic tissues: a quantitative study. Biophysical journal. 1998; 74(5):2227-2234. [PubMed: 9591650]

[7]. Kuznetsova TG, Starodubtseva MN, Yegorenkov NI, Chizhik SA, Zhdanov RI. Atomic force microscopy probing of cell elasticity. Micron. 2007; 38(8):824-833. [PubMed: 17709250]

[8]. Cheng G, Tse J, Jain RK, Munn LL. Micro-environmental mechanical stress controls tumor spheroid size and morphology by suppressing proliferation and inducing apoptosis in cancer cells. PLoS one. 2009; 4(2):e4632. [PubMed: 19247489]

[9]. Netti PA, Berk DA, Swartz MA, Grodzinsky AJ, Jain RK. Role of extracellular matrix assembly in interstitial transport in solid tumors. Cancer research. 2000; 60(9):2497-2503. [PubMed: 10811131]

[10]. Stylianopoulos T, Martin JD, Chauhan VP, Jain SR, Diop-Frimpong B, Bardeesy N, Smith BL, Ferrone CR, Hornicek FJ, Boucher Y, et al. Causes, consequences, and remedies for growthinduced solid stress in murine and human tumors. Proceedings of the National Academy of Sciences. 2012; 109(38):15 101-15 108.

[11]. Ambrosi D, Mollica F. On the mechanics of a growing tumor. International journal of engineering science. 2002; 40(12):1297-1316.

[12]. Montel F, Delarue M, Elgeti J, Malaquin L, Basan M, Risler T, Cabane B, Vignjevic D, Prost J, Cappello G, et al. Stress clamp experiments on multicellular tumor spheroids. Physical review letters. 2011; 107(18):188 102.

[13]. Monnier S, Delarue M, Brunel B, Dolega ME, Delon A, Cappello G. Effect of an osmotic stress on multicellular aggregates. Methods. 2016; 94:114-119. [PubMed: 26210402]

[14]. Byrne H, Preziosi L. Modelling solid tumour growth using the theory of mixtures. Mathematical Medicine and Biology. 2003; 20(4):341-366. [PubMed: 14969384]

[15]. Ambrosi D, Preziosi L. On the closure of mass balance models for tumor growth. Mathematical Models and Methods in Applied Sciences. 2002; 12(05):737-754.

[16]. Roose T, Netti PA, Munn LL, Boucher Y, Jain RK. Solid stress generated by spheroid growth estimated using a linear poroelasticity model. Microvascular research. 2003; 66(3):204-212. [PubMed: 14609526]

[17]. Ambrosi D, Guana F. Stress-modulated growth. Mathematics and mechanics of solids. 2007; 12(3):319-342. 
[18]. Chanet S, Martin AC. Mechanical force sensing in tissues. Progress in molecular biology and translational science. 2014; 126:317. [PubMed: 25081624]

[19]. Ciarletta P. Buckling instability in growing tumor spheroids. Physical review letters. 2013; 110(15):158 102.

[20]. Destrade M, Murphy JG, Ogden RW. On deforming a sector of a circular cylindrical tube into an intact tube: existence, uniqueness, and stability. International Journal of Engineering Science. 2010; 48(11):1212-1224.

[21]. Alnæs M, Blechta J, Hake J, Johansson A, Kehlet B, Logg A, Richardson C, Ring J, Rognes ME, Wells GN. The fenics project version 1.5. Archive of Numerical Software. 2015; 3(100)

[22]. Geuzaine C, Remacle JF. Gmsh: A 3-d finite element mesh generator with built-in pre-and postprocessing facilities. International Journal for Numerical Methods in Engineering. 2009; 79(11): 1309-1331.

[23]. Balay, S., Abhyankar, S., Adams, MF., Brown, J., Brune, P., Buschelman, K., Dalcin, L., Eijkhout, V., Gropp, WD., Kaushik, D., et al. PETSc users manual. Technical Report ANL-95/11 - Revision 3.6. Argonne National Laboratory; 2015.

[24]. Amestoy PR, Duff IS, L'Excellent JY, Koster J. A fully asynchronous multifrontal solver using distributed dynamic scheduling. SIAM Journal on Matrix Analysis and Applications. 2001; 23(1):15-41.

[25]. Mascheroni P, Stigliano C, Carfagna M, Boso DP, Preziosi L, Decuzzi P, Schrefler BA. Predicting the growth of glioblastoma multiforme spheroids using a multiphase porous media model. Biomechanics and modeling in mechanobiology. 2016:1-14.

[26]. Ambrosi D, Preziosi L. Cell adhesion mechanisms and stress relaxation in the mechanics of tumours. Biomechanics and modeling in mechanobiology. 2009; 8(5):397-413. [PubMed: 19115069]

[27]. Dorie MJ, Kallman RF, Rapacchietta DF, Van Antwerp D, Huang YR. Migration and internalization of cells and polystyrene microspheres in tumor cell spheroids. Experimental cell research. 1982; 141(1):201-209. [PubMed: 7117414]

[28]. Delarue M, Montel F, Caen O, Elgeti J, Siaugue JM, Vignjevic D, Prost J, Joanny JF, Cappello G. Mechanical control of cell flow in multicellular spheroids. Physical review letters. 2013; 110(13): 138103. 


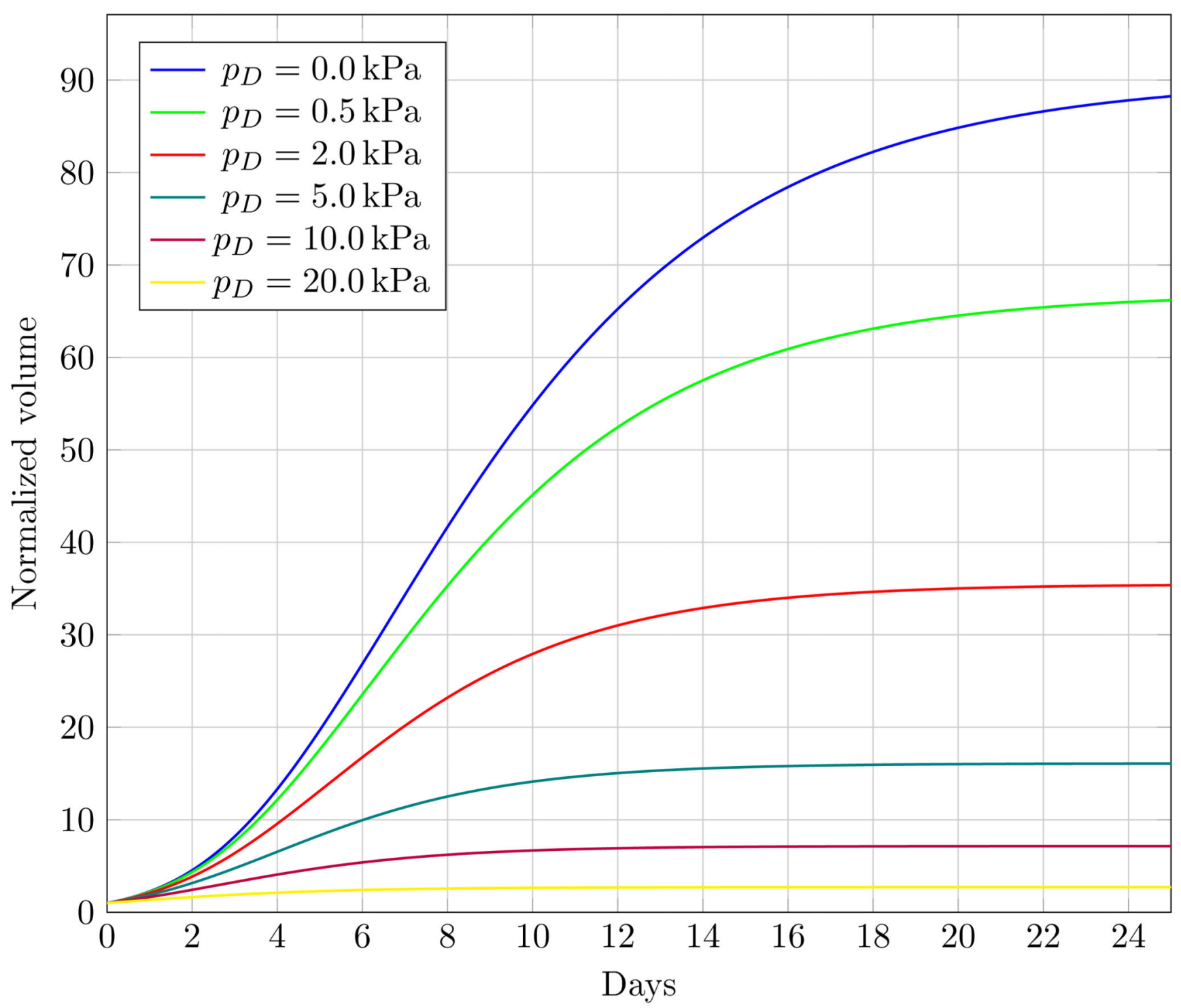

Figure 1.

Volume of a spheroid vs. time for different values of the osmotic pressure. 


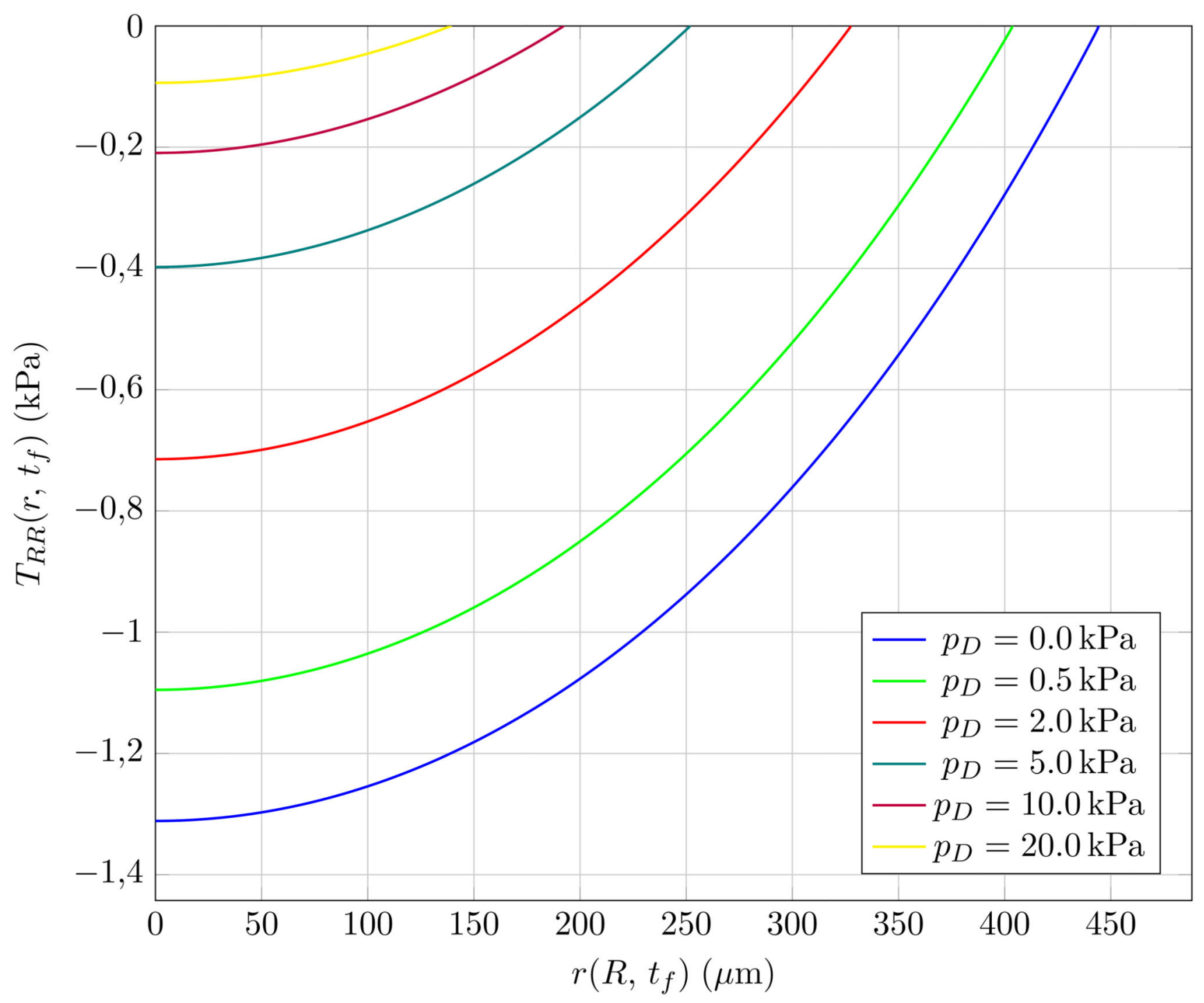

Figure 2.

Radial residual stress versus the radial position at final time for different values of the osmotic pressure. 


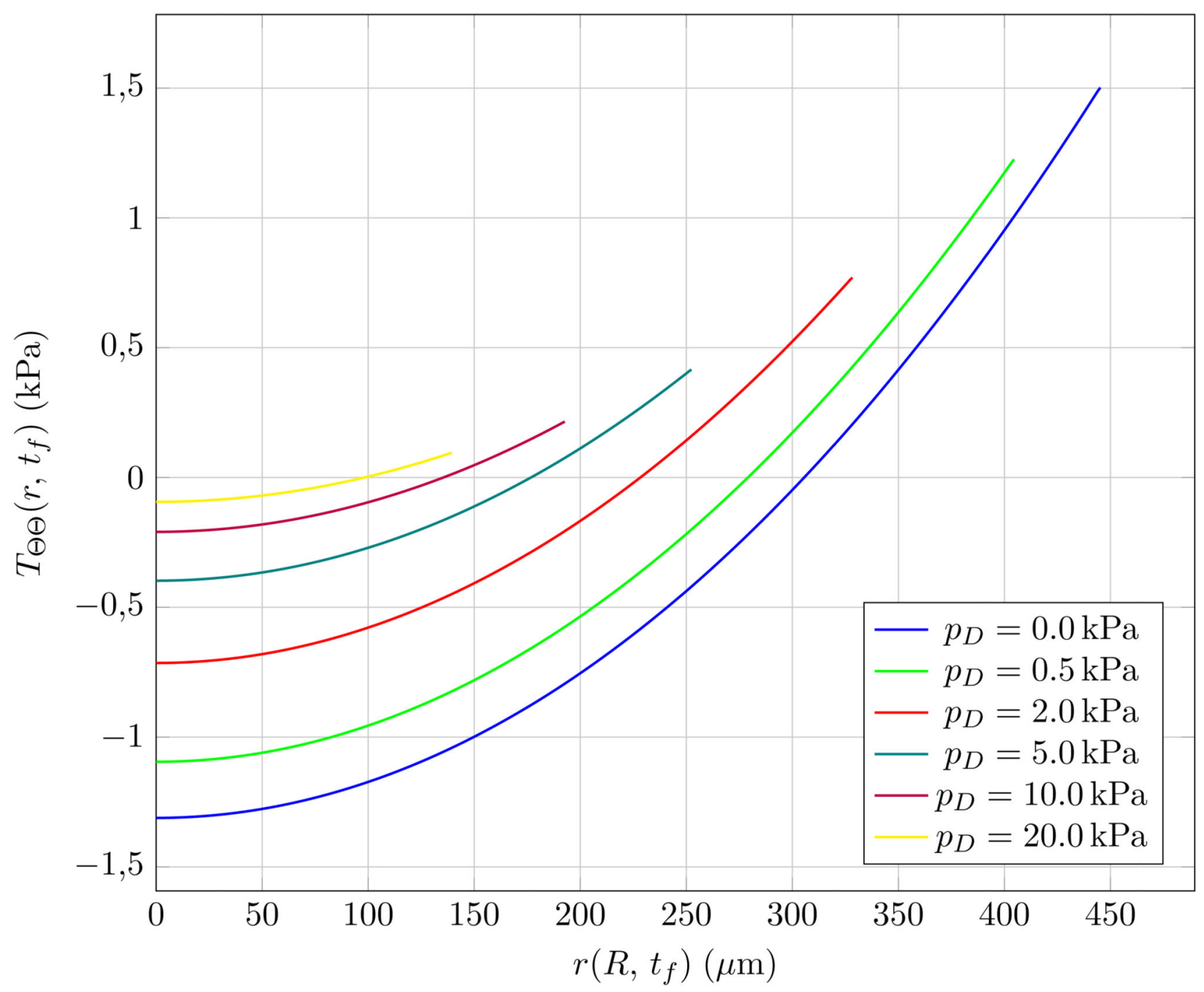

Figure 3.

Hoop residual stress versus the radial coordinate at final time for different values of the osmotic pressure. 


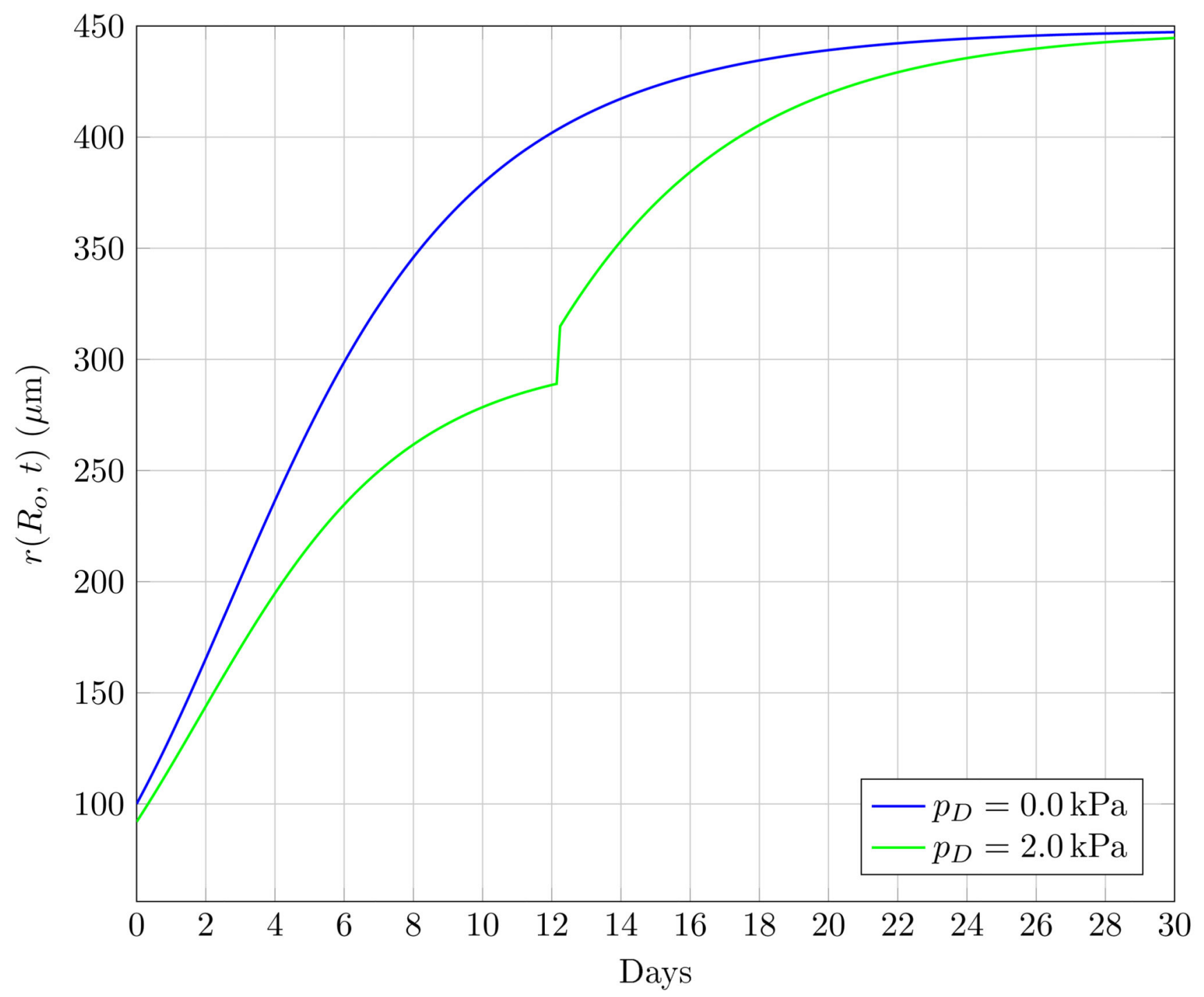

Figure 4.

Radius of the spheroid vs. time for $p_{D}=0$ (blue line) and $p_{D}=2000 \mathrm{~Pa}$ (green line). After 12 days the external pressure is removed and the system returns the curve corresponding to the unloaded state. 


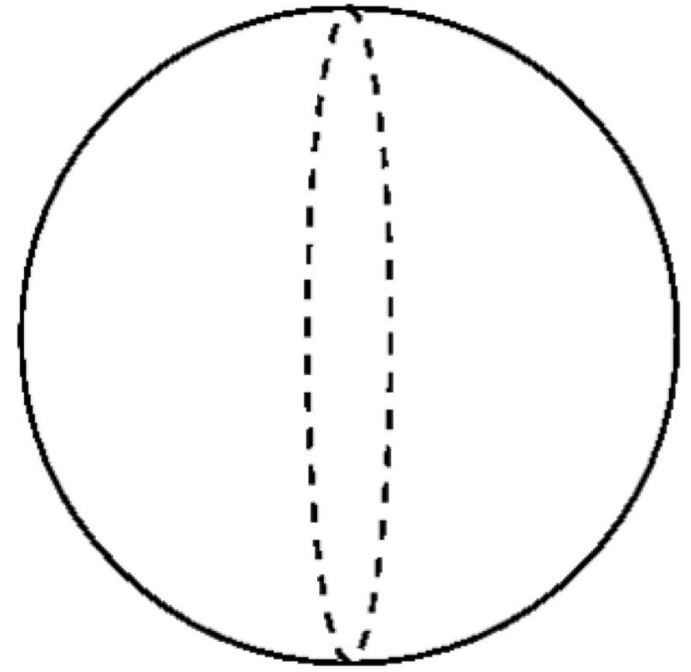

intact tumor

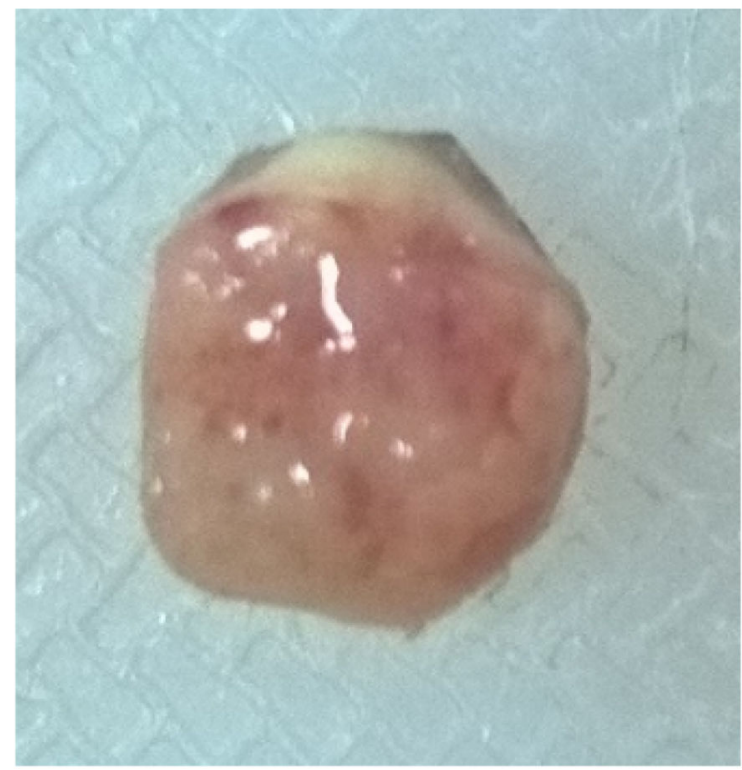

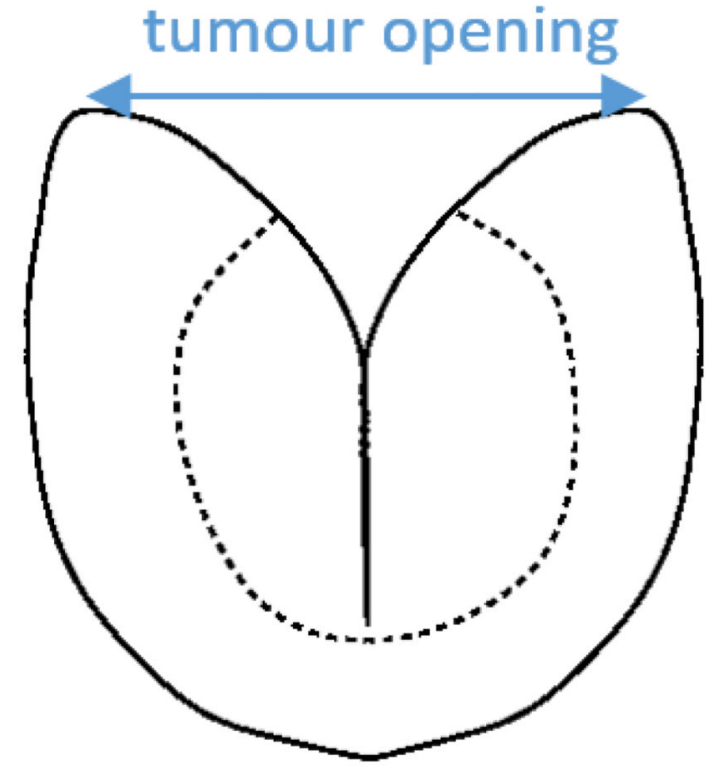

azimuthally cut tumor

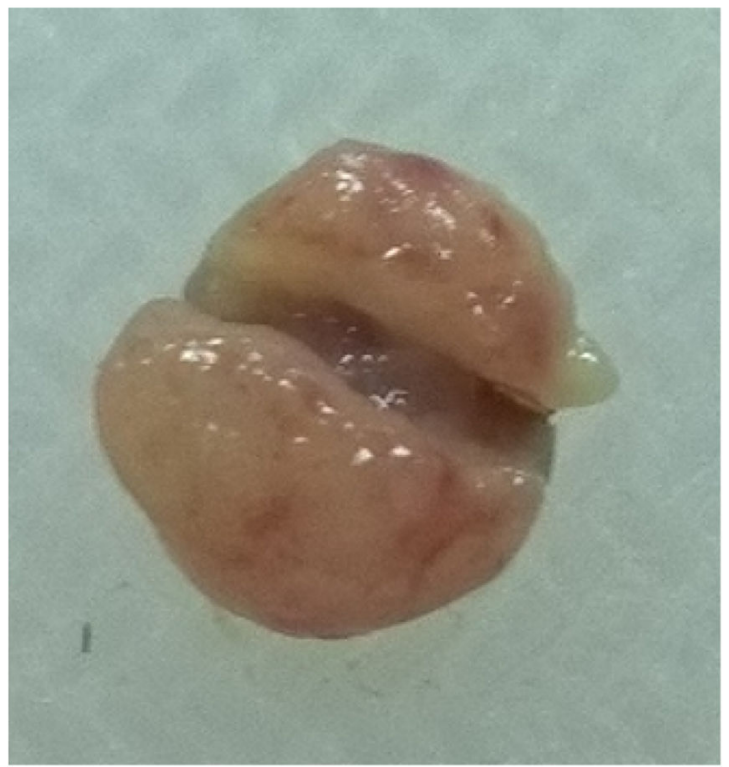

Figure 5.

An intact, residually stressed spheroid (left) is cut along an azimuthal plane for $80 \%$ of its diameter. The outer region opens up while the inner one swells (right), thus distribution of residual stress that goes from compressive to tensile along the radial coordinate. 


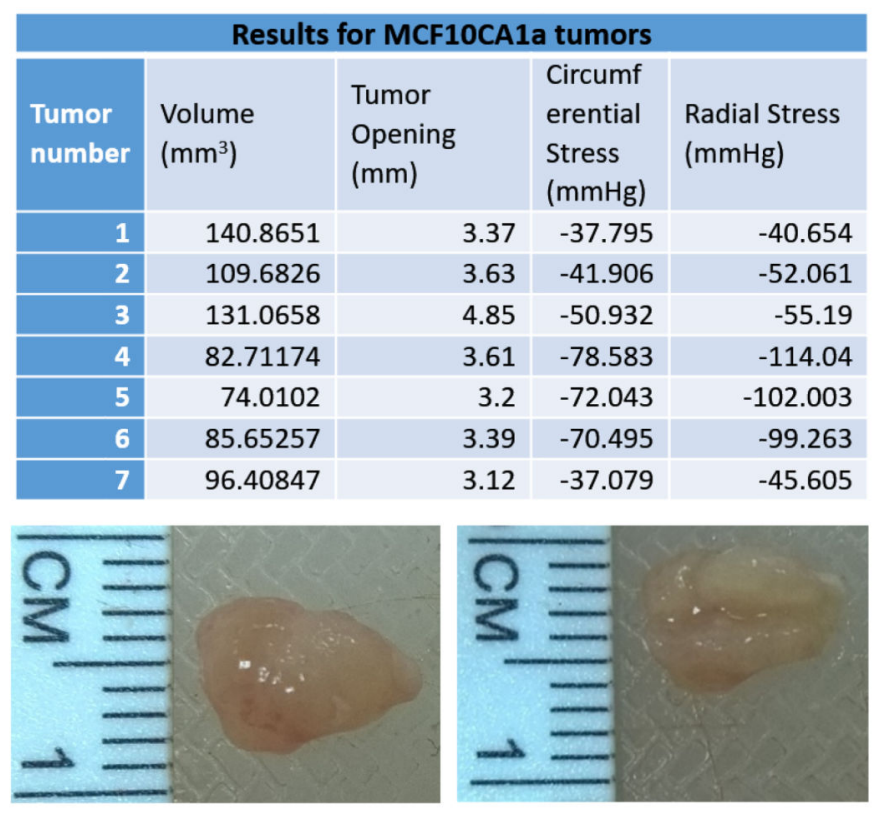

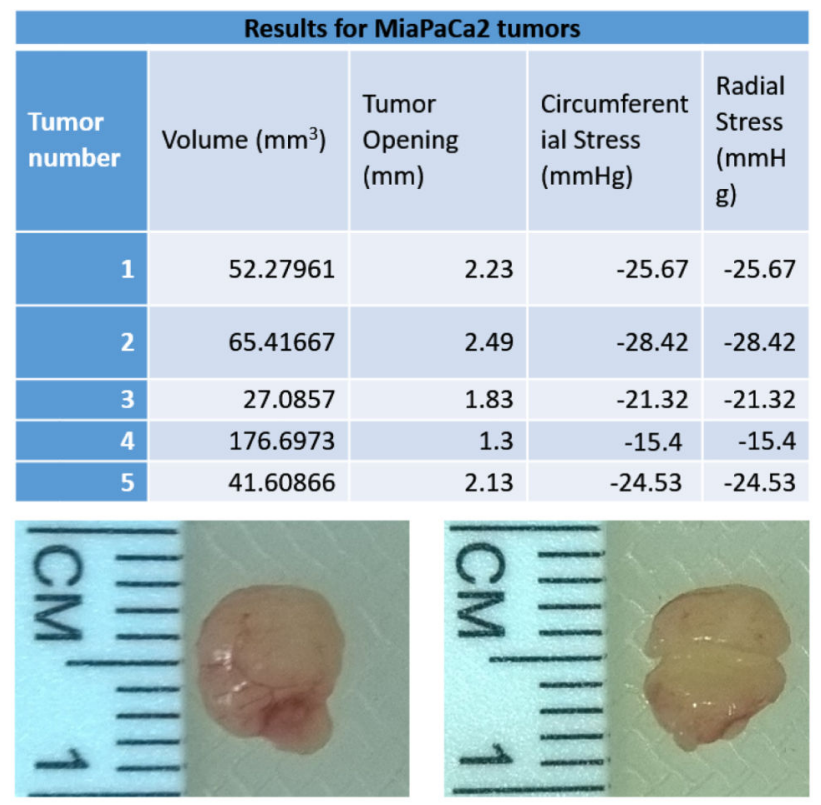

Figure 6.

Tables with experimental measures (top) of cutting experiments (bottom) for MCFIOCAla breast tumor cells (left) and for MiaPaCa2 pancreatic tumor cells (right) implanted orthotopicallv in the mammary fat pad of nude mice. 


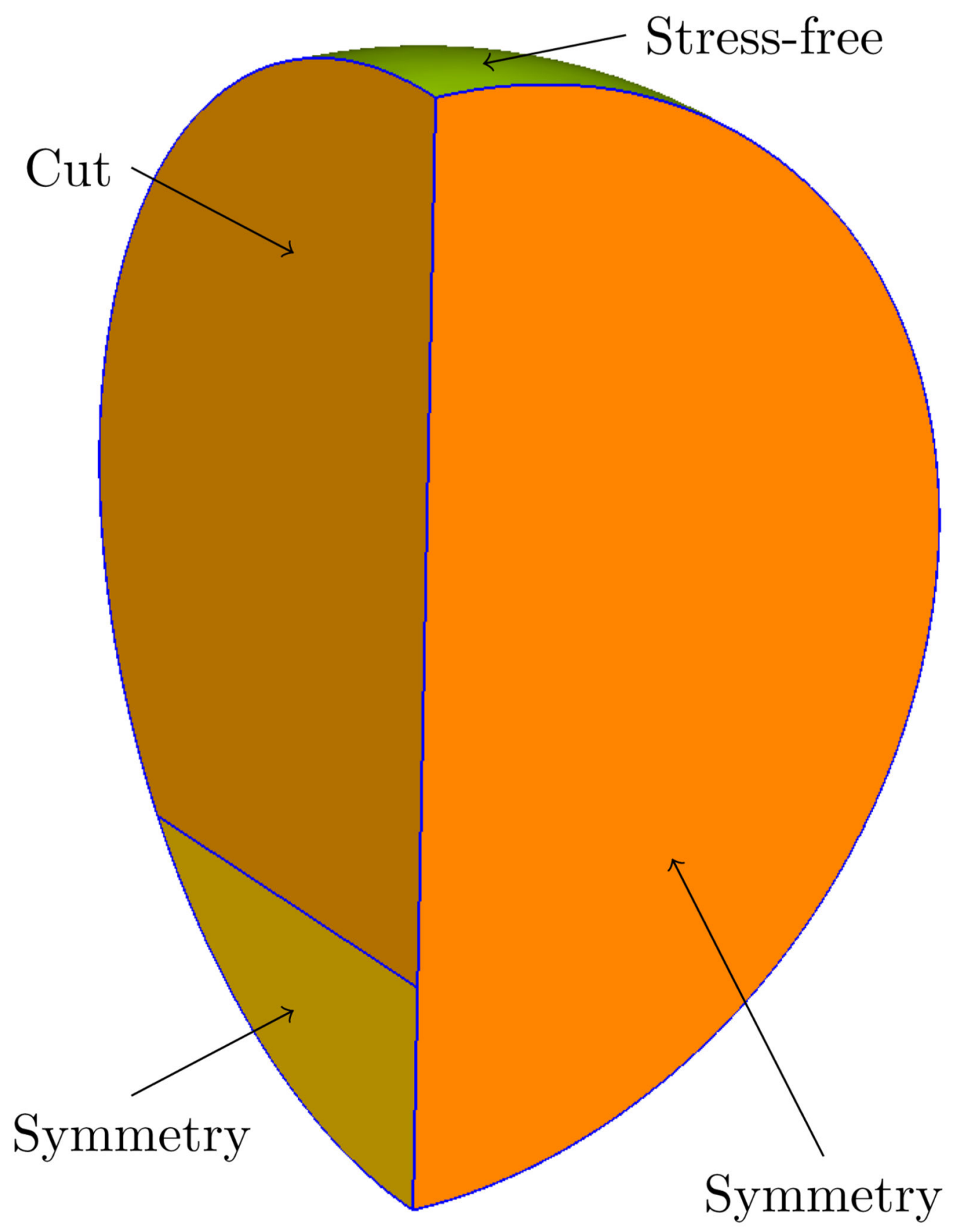

Figure 7.

Computational domain and boundary conditions for the $3 \mathrm{~d}$ numerical experiment of the opening angle. The "symmetry" label indicates that the surface is fixed in the normal direction. The vertical cut is $80 \%$ of the diameter deep. 


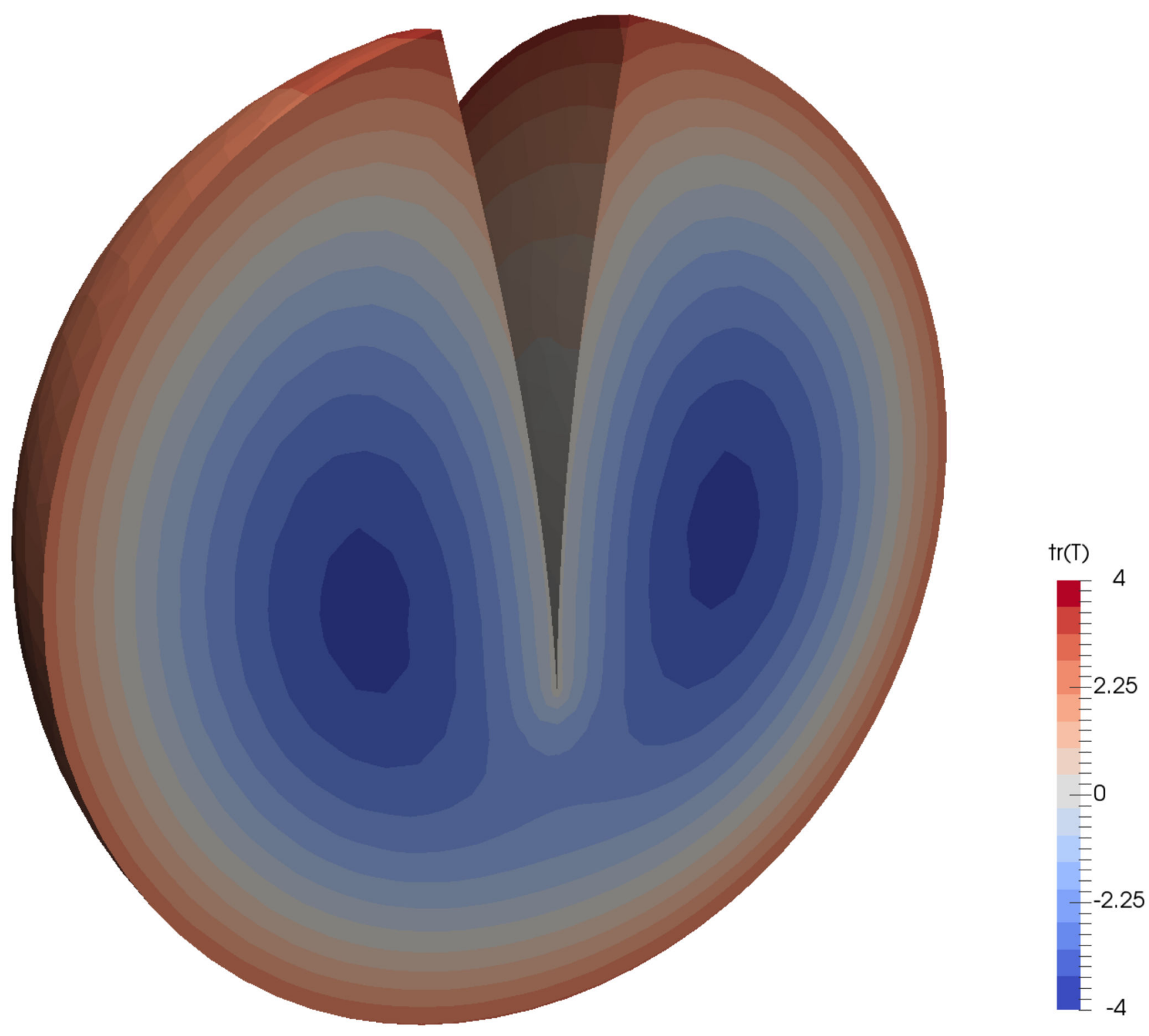

Figure 8.

Current configuration of an unloaded grown sphere with initial radius of $100 \mu \mathrm{m}$. The sphere is azimuthally cut at the final time. The parameters in the simulation are: $\tau=2.5$ days, $\kappa=$ $33.35, \alpha=37, \lambda=2.5 \mathrm{~mm}, \mu=27.0 \mathrm{kPa}$ and $p_{D}=5.0 \mathrm{kPa}$. The domain of the numerical simulation is a quarter of a sphere. For the sake of graphical clarity of the opening angle, the spherical wedge is combined with its symmetric counterpart. The body is unloaded but not stress free: the color map represents the trace of the Cauchv stress tensor. The lowest part of the cut is partially resew by the swelling. 


\section{Table 1}

Numerical result of a stepwise increase of the difference in growth between the center and the boundary of the tumor. The growth function $g(R)$ is linear in the radial component.

\begin{tabular}{|l|l|l|l|l|l|l|}
\hline$g(R=0)$ & 45.0 & 44.0 & 43.0 & 42.0 & 41.0 & 40.0 \\
\hline$g(R=R 0)$ & 45.0 & 48.0 & 51.0 & 54.0 & 57.0 & 60.0 \\
\hline opening length $[\mathrm{mm}]$ & 0.0 & 0.540 & 1.12 & 1.70 & 2.30 & 2.92 \\
\hline opening angle $[\mathrm{deg}]$ & 0.0 & 3.43 & 6.94 & 10.5 & 14.2 & 18.0 \\
\hline volume $\left[\mathrm{mm}^{3}\right]$ & 94.7 & 94.8 & 95.0 & 95.4 & 95.9 & 96.6 \\
\hline
\end{tabular}

